BMJ Paediatrics Open

\title{
Is arterial stiffening associated with adiposity, severity of obesity and other contemporary cardiometabolic markers in a community sample of adolescents with obesity in the UK?
}

\author{
Lee Hudson, ${ }^{1}$ Sanjay Kinra, ${ }^{2}$ lan Wong, ${ }^{3}$ Tim J Cole, ${ }^{1}$ John Deanfield, ${ }^{1}$ \\ Russell Viner $^{1}$
}

To cite: Hudson L, Kinra S, Wong I, et al. Is arterial stiffening associated with adiposity, severity of obesity and other contemporary cardiometabolic markers in a community sample of adolescents with obesity in the UK? BMJ Paediatrics Open 2017;1:e000061. doi:10.1136/ bmjpo-2017-000061

- Prepublication history for this paper is available online. To view these files please visit the journal online (http://dx.doi.org/ 10.1136/bmjpo-2017-000061).

Received 2 May 2017 Revised 27 June 2017 Accepted 29 June 2017

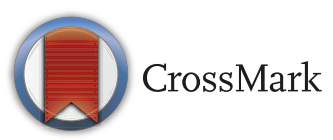

${ }^{1}$ UCL Great Ormond Street Institute of Child Health, London, UK

${ }^{2}$ London School of Hygiene and Tropical Medicine, London, UK ${ }^{3} \mathrm{UCL}$ School of Pharmacy, London, UK

\section{Correspondence to} Dr Lee Hudson; I.hudson@ucl. ac.uk

\section{ABSTRACT}

Background Cardiovascular disease (CVD) prediction is problematic within groups of obese adolescents as measures such as adiposity and metabolic markers lack validation. Pulse wave velocity (PWV), a proxy for arterial stiffening, is a potential way to contemporaneously capture adolescents at greater risk of CVD.

Objectives To investigate associations between PWV and 1) adiposity and 2) other conventional metabolic factors in a community sample of ( $>95$ th centile body mass index (BMI)).

Design and setting Cross-sectional measurement and analysis in a hospital-based research centre drawn from a community sample of adolescents recruited to an obesity intervention at baseline.

Patients 174 adolescents (12-19 years) with obesity (>95th centile BMI). 37\% were male, while $66(38 \%)$ were white, 53 (30\%) black, 36 (21\%) South Asian, 19 (11\%) mixed/other. Participants with endocrine, genetic causes of obesity and chronic medical conditions (excluding asthma) were excluded.

Measures BMI z-score (zBMI), waist z-score, fat mass index (FMl: measured using bioimpedance), sagittal abdominal dimension (SAD), cardiometabolic blood tests and resting blood pressure (BP) were collected. Carotidradial PWV was measured by a single operator. Results PWV was associated with age but not pubertal stage. PWV was positively associated with adiposity (zBMl: coefficient 0.44 ( $95 \% \mathrm{Cl} 0.08$ to 0.79 ); FMl: coefficient 0.05 (95\% Cl 0.00 to 0.10); waist z-score: coefficient 0.27 (95\% Cl 0.00 to 0.53 ); SAD: coefficient 0.06 (95\% Cl: 0.00 to 0.12$)$ ). There was no association between PWV and $\mathrm{BP}$, and few associations with cardiometabolic bloods. Associations between PWV and adiposity measures were robust to adjustment in multivariable models except for SAD. Participants with zBMI >2.5 SD and >3.5 SD had greater average PWV but overlap between groups was large. Conclusions In our sample, increasing adiposity was positively associated with arterial stiffness, however partitioning by severity was not reliable. Lack of associations between BP, cardiometabolic bloods and arterial stiffness questions the reliability of these factors for predicting CVD risk in adolescents with obesity.

\section{What is already known on this topic?}

Pulse wave velocity (PWV) has been shown to be greater in children with obesity compared with healthy weight controls.

- Validity of degree of adiposity, metabolic blood markers and blood pressure as predictors of longterm cardiovascular disease within obese groups of adolescents is unclear.

\section{What this study adds?}

- Adiposity was associated with PWV yet overlap of distributions when partitioned by obesity severity calls into question what constitutes 'severe obesity' for cardiovascular risk.

- PWV was not associated with blood pressure and with few other metabolic markers.

\section{INTRODUCTION}

Rising obesity rates among children and young people (CYP) have presented the new challenge of identifying which groups, and which patients in the clinical setting, require focus for interventions for both weight loss programmes and possible active medical management. ${ }^{12}$ Current medical management algorithms for CYP obesity use severity of obesity (based on body mass index (BMI) classification) and family history as proxies for cardiovascular disease (CVD) risk to classify individuals for further assessment and treatment. ${ }^{3}{ }^{4}$ However, conventional cut-offs to define obesity in childhood ${ }^{5}$ (such as above the 95th centile, ${ }^{4}$ International Obesity Task Force cut-offs ${ }^{6}$ and $>2.5 \mathrm{SDs}^{7}$ and $>3.5 \mathrm{SD}$ for severe obesity ${ }^{3}$ ) currently lack validation as predictors of long-term CVD risk. Published studies examining the usefulness of cut-offs 
have focused on their ability to capture components of the 'metabolic syndrome', that is, established adult CVD risk factors such as hypertension and dyslipidemia, ${ }^{7-9}$ as well as reporting the tracking of children defined with obesity into adulthood. ${ }^{10}{ }^{11}$ Evidence to support the importance of such metabolic risk factors as predictors of future CVD risk in children, either individually or in combination, ${ }^{12-14}$ is also limited; and their persistence may be temporally unstable through adolescence. ${ }^{15}$ Predicting CVD risk in young people with obesity therefore remains problematic, especially in the clinical setting for individual patients. ${ }^{16} 17$

Another approach for exploring current CVD risk in adolescents with obesity is to seek contemporaneous evidence of arterial stiffening, ${ }^{18} 19$ with pulse wave velocity (PWV) being a potentially useful and non-invasive proxy. ${ }^{20-22}$ A derivative gradient velocity calculated from pulse waveforms at two separate peripheral arterial loci and the distance between them (with PWV proportional to arterial stiffness), PWV is reproducible, ${ }^{22}{ }^{23}$ correlates with arterial plaque load in adults ${ }^{24}$ and accurately predicts CVD mortality in adults. ${ }^{2526}$ Two meta-analyses have demonstrated that CYP with obesity have greater PWV compared with non-obese controls. ${ }^{27}{ }^{28}$ Studies have examined the relationship between adiposity and PWV at various anatomical sites in children and adolescents combining healthy and overweight status with variable findings. ${ }^{29-36}$ However, the majority of these studies have not used standardised measures of adiposity (ie, z-scores of BMI and waist circumference), and have not adjusted for other potential key factors such as puberty and ethnicity. ${ }^{18}$ Such information is important when considering CVD risk for patients within obese groups of CYP.

We used data from a cross-sectional, community sample of adolescents with obesity recruited into an obesity intervention trial to examine the associations of contemporary arterial stiffening, using PWV, with multiple measures of adiposity, conventional markers of metabolic risk (blood pressure (BP) and cardiometabolic blood markers) and pubertal status and ethnicity. We hypothesised that arterial stiffness would increase with degree of adiposity and would be positively associated with other cardiovascular risk factors, in particular BP. We also examined whether different thresholds, commonly used to partition groups of obese CYP for defining severe obesity, were associated with PWV differently.

\section{MATERIALS AND METHODS}

Data were obtained from the baseline sample of the Healthy Eating and Lifestyle Trial (HELP) trial, a randomised controlled trial of an adolescent obesity intervention. ${ }^{37}$ Adolescents aged 12-19 years were recruited from community sources (including general practitioners, schools, youth groups and self referral) in the Greater London area between January 2011 and July 2013. Individuals with chronic illnesses (excluding asthma or more minor chronic conditions such as eczema), genetic or endocrine causes of obesity and those with diagnosed mental health problems were excluded. Participants were assessed at the Clinical Research Facility at Great Ormond Street Hospital at baseline (before randomisation) by a physician. Trained nurses collected the data using standardised protocols and the same equipment throughout. Ethics permission for the study was provided by the regional, central London ethics committee.

Ethnicity was self-reported and categorised as white, black, South Asian or mixed/other ethnicity. Participants were asked to self-report their pubertal status using standardised diagrams ${ }^{38}$ and report of menarcheal status in girls. Pubertal status was then categorised as pre/early (Tanner stages 1 and 2), mid (Tanner 3 and 4), late/complete (Tanner stage 5). Girls postmenarche were grouped as late/complete by default. Self-report data on smoking were provided by young people, with participants grouped into binary groups in two ways: 1) as 'current smokers' (ie, current vs non-smoker or ex-smoker) and 2) 'ever smoked' (current and ex-smoker vs non-smoker).

\section{Anthropometry}

Heights were measured to the nearest $0.1 \mathrm{~cm}$ using an electronic stadiometer (Seca 242 Electronic Measuring Rod, Seca GmbH, Germany). Weight and 4-limb bioimpedence were measured to the nearest $0.01 \mathrm{~kg}$ on the Tanita BC 418MA (Tanita, UK), in loose clothing. Total impedance was used to calculate fat mass $(\mathrm{kg})$ using a validated formula for use in adolescents with obesity. ${ }^{39}$ Waist circumference was measured midway between the 10th rib and iliac crest to the nearest $\mathrm{mm}$ using a non-elastic flexible tape in the standing position, ${ }^{40}$ with values averaged over three measurements. Anterior-posterior sagittal abdominal dimension (SAD) was measured to the nearest $0.1 \mathrm{~cm}$ by calliper (Holtain Kahn abdominal calliper, Holtain, UK) at the centre of the abdomen following exhalation with participants in a supine position. ${ }^{41}$ BMI was calculated as weight $(\mathrm{kg}) /$ height $(\mathrm{m})^{2}$, and fat mass index (FMI) was calculated as fat mass $(\mathrm{kg}) /$ height $(\mathrm{m})^{2}$. Standardised BMI and waist circumference z-scores (zBMI and waist-z) were calculated using the LMSgrowth program V.2.69 (Harlow Healthcare, UK) using the UK 1990 growth reference data. ${ }^{42}{ }^{43}$ Raw data were used for FMI and SAD as no validated standardised data are available. Obesity status was categorised using two cut-offs for severe obesity: $\mathrm{zBMI}>2.5^{8}$ and $\mathrm{zBMI}>3.5$. $^{3}$

\section{Blood pressure}

BP was measured after 20 min seated rest using an automated machine (Philiips IntelliVue MP30 Monitor, Koninklijke Phillips N.V., Holland). BP was taken at the right arm three times serially, with the third value recorded. Systolic and diastolic BP were converted to z-scores using LMSgrowth program V.2.69 (Harlow Healthcare), which uses the UK BP population data. ${ }^{43} 44$ Hypertension was defined as either systolic or diastolic $\mathrm{BP}>98$ th centile (2 SD above the mean). ${ }^{3}$ 


\section{Blood parameters}

Blood was drawn following a 10-12 hours fast. Bloods were analysed for cholesterol (including high-density lipoprotein (HDL)), triglycerides, glucose, \% glycated haemoglobin, insulin and alanine aminotransferase (ALT). Blood was processed at Great Ormond Street Hospital laboratory. Homeostatic model assessment-insulin resistance (HOMA-IR) was derived using formula (insulinxglucose/2). Abnormality flags were generated for individual blood variables using cut-offs based on a UK consensus statement, ${ }^{3}$ as follows: 'abnormal HOMA-IR' > 4.4, 'raised insulin' for insulin levels above values based on pubertal stage $(>10 \mathrm{mU} / \mathrm{L}$ prepuberty/early puberty, $>30 \mathrm{mU} / \mathrm{L}$ mid-puberty, $>20 \mathrm{mU} / \mathrm{L}$ late and complete puberty), 'low HDL' $0.9 \mathrm{mmol} / \mathrm{L}$, 'raised triglycerides' $>1.47 \mathrm{mmol} / \mathrm{L}$, 'raised cholesterol' $>5.2 \mathrm{mmol} / \mathrm{L}$, 'abnormal HDL/C ratio' $>4.3$.

\section{Measurement of PWV}

Patients in a fasted state rested for $30 \mathrm{~min}$ prior to measurement at room temperature, supine. Pulse waves were measured at the carotid and radial pulses using tonometry and appropriate software (SphygmoCor, AtCor Medical, Sydney, Australia) by a single operator for all participants. The distance between the carotid and sternal notch, and the sternal notch to the radial point (via the mid-shoulder) were also measured, and combined with waveforms and peripheral BPs (as above) by software to derive carotid-radial PWV values. Data were recorded only once per participant, and only recorded readings met quality control measures according to the manufacturer's protocol (SD of derived $\mathrm{PWV}<10 \%$ of the $\mathrm{PWV}$ value, all $\mathrm{PWV}$ wave forms within window and timing $\mathrm{SD}<6 \%$ ).

\section{Analysis}

Data were managed and analysed using Stata V.13 (StataCorp, Texas, USA). Data were cleaned and individual variables examined for normality. Participant characteristics were described using mean and SD for normally distributed continuous variables, and median and IQR otherwise. Group means were compared using t-test or $\chi^{2}$ test for normally distributed variables, and non-parametric tests otherwise. Group proportions were compared using $\chi^{2}$ test. Associations of anthropometric, metabolic risk factors, demographic variables and PWV were investigated using linear regression. Individual variables found to be associated with PWV at $\mathrm{p}<0.1$ were entered into multivariable regression models. As zBMI, waist-z, FMI and SAD are recognised as proxies for adiposity (while also independently associated), each was entered into univariable and multivariable regression models discretely. Non-normally distributed continuous variables were transformed for use in univariable regression analysis. If the transformation did not alter the models significantly, models using non-transformed variables were presented. Effect sizes of models were described using coefficients of determination ( $\mathrm{R}^{2}$ for univariable and multivariable models, with small, medium and large effects defined conventionally as $\mathrm{R}^{2}>0.02,>0.13$ and $>0.26$, respectively. ${ }^{45}$
RESULTS

A total of 174 children were recruited to the HELP trial (65 $(37 \%)$ male). Proportions in each ethnic group were: white $66(38 \%)$, black $53(30 \%)$, South Asian $36(21 \%)$, mixed/other $19(11 \%)$. Proportions at each pubertal stage were pre/early (Tanner $1 / 2) 21(12 \%)$, mid $(3 / 4)$ $38(22 \%)$, late/post (5) $115(66 \%)$. There were more females in complete/late puberty than in other stages ( $89 \%$ vs $12 \%, \mathrm{p}<0.001)$. The sex ratio was similar in all ethnic groups. Fourteen (8\%) participants were current smokers and 48 (29\%) were either current or ex-smokers.

Characteristics of continuous variables for the study group are shown in table 1 . Age was not normally distributed, but the sample appeared evenly distributed across age groups. Males were taller than females but similar in height z-score, while females had greater waist-z and FMI. Males had greater metabolic markers as triglycerides, fasting glucose and ALT; 126 participants (72\%) had zBMI $>2.5$ and $17(10 \%)$ exceeded 3.5.

\section{Metabolic risk factors}

No participants had systolic hypertension or evidence of impaired fasting glucose. Four (2\%) had diastolic hypertension, $35(20 \%)$ raised HOMA, 34 (20\%) raised insulin for pubertal stage, 29 (17\%) elevated cholesterol, $29(17 \%)$ raised triglycerides, 20 (12\%) low HDL and 66 $(39 \%)$ had an elevated $\mathrm{HDL} / \mathrm{C}$ ratio.

\section{Pulse wave analysis}

Pulse wave data were available for 146 (84\%) participants. Univariable regression analyses between PWV and anthropometric, cardiometabolic and demographic markers are shown in table 2. Significant positive associations were found between PWV and age, all adiposity markers (zBMI, FMI, waist-z and SAD), and abnormal triglyceride grouping (effects sizes were however small, $\mathrm{R}^{2}=0.05,0.04$, 0.03, 0.03, 0.03, 0.02, respectively). Ethnic South Asians, and to a lesser extent blacks, had higher PWV compared with ethnic whites. PWV was lower in the low HDL group. Abnormal triglyceride grouping was no longer significant once adjusted for age, ethnicity and zBMI (data not shown). Low HDL grouping remained negatively associated with PWV when controlled for zBMI, age, ethnicity group (coefficient -0.60 (95\% CI -1.17 to -0.03 ), beta $\left.-0.17, \mathrm{p}=0.04, \mathrm{r}^{2} 0.17\right)$. There were no differences in PWV by sex, pubertal stage, smoking or cardiometabolic markers (including systolic and diastolic BP). Scatter plots of PWV versus measures of adiposity, with fitted regression lines, are shown in in figure 1. In general the associations are weak.

Multivariable regression models for each adiposity measure adjusting for age, ethnicity, abnormal triglycerides and abnormally low HDL are shown in table 3. There was a positive association between PWV and all adiposity measures except SAD. Effect sizes for adjusted models for each adiposity measure were medium in size $\left(\mathrm{R}^{2}\right.$ in adjusted models for $\mathrm{zBMI}=0.16$, waist-Z=0.13 and $\mathrm{FMI}=0.15)$. 
Table 1 Summary of continuous variables by sex

\begin{tabular}{|c|c|c|c|c|c|c|c|c|c|c|c|c|c|c|c|}
\hline \multirow[b]{2}{*}{ Variable } & \multicolumn{5}{|c|}{ Males } & \multicolumn{5}{|c|}{ Females } & \multicolumn{5}{|c|}{ All subjects } \\
\hline & $\mathbf{n}$ & Mean & Median & SD & IQR & n & Mean & Median & SD & IQR & $\bar{n}$ & Mean & Median & SD & IQR \\
\hline Age (years) & 65 & & 15.0 & & 3.2 & 109 & & 15.6 & & 2.9 & 174 & & 15.3 & & 3.2 \\
\hline Weight (kg) & 65 & & 87.4 & & 23 & 109 & & 85.4 & & 20.6 & 174 & & 86.2 & & 22 \\
\hline Height (cm) & 65 & $168.4^{*}$ & & 9.6 & 13.2 & 109 & 164.3 & & 6.5 & & 174 & 165.8 & & 8.1 & \\
\hline Height z & 65 & 0.39 & & 0.88 & & 109 & 0.52 & & 1.00 & & 174 & 0.47 & & 0.96 & \\
\hline BMI $\left(\mathrm{kg} / \mathrm{m}^{2}\right)$ & 65 & & 30.9 & & 5.9 & 109 & & 32.5 & & 6.1 & 174 & & 32 & & 6.1 \\
\hline zBMI & 65 & 2.83 & & 0.47 & & 109 & 2.78 & & 0.59 & & 174 & 2.80 & & 0.55 & \\
\hline Waist $(\mathrm{cm})$ & 65 & & 101 & & 15 & 109 & & 95.4 & & 17 & 174 & & 99 & & 15.9 \\
\hline Waist-z & 65 & $2.97^{*}$ & & 0.51 & & 109 & 3.68 & & 0.68 & & 174 & 3.45 & & 0.72 & \\
\hline $\mathrm{SAD}(\mathrm{cm})$ & 57 & 23.1 & & 3.2 & & 102 & 22.3 & & 3.5 & & 159 & 22.6 & & 3.4 & \\
\hline FMI $\left(\mathrm{kg} / \mathrm{m}^{2}\right)$ & 63 & $12.5^{\star}$ & & 4.0 & & 108 & 15.0 & & 3.6 & & 171 & 14.1 & & 3.89 & \\
\hline $\begin{array}{l}\text { Systolic BP } \\
(\mathrm{mm} \mathrm{Hg})\end{array}$ & 63 & 109 & & 10 & & 109 & 106 & & 10. & & 172 & 107 & & 10 & \\
\hline Systolic BP z & 63 & -1.05 & & 1.03 & & 109 & -1.09 & & 1.08 & & 172 & -1.07 & & 1.06 & \\
\hline $\begin{array}{l}\text { Diastolic BP } \\
(\mathrm{mm} \mathrm{Hg})\end{array}$ & 63 & 53 & & 9 & & 109 & 54 & & 10 & & 172 & 54 & & 9 & \\
\hline $\begin{array}{l}\text { Diastolic } \\
\text { BP z }\end{array}$ & 63 & -0.60 & & 1.10 & & 109 & -0.63 & & 1.19 & & 172 & -0.61 & & 1.15 & \\
\hline $\begin{array}{l}\text { Cholesterol } \\
(\mathrm{mmol} / \mathrm{L})\end{array}$ & 65 & 4.5 & & 0.9 & & 109 & 4.3 & & 0.8 & & 174 & 4.4 & & 0.8 & \\
\hline $\begin{array}{l}\text { Triglycerides } \\
\text { (mmol/L) }\end{array}$ & 64 & & $1.1^{*}$ & & 0.6 & 109 & & 0.9 & & 0.6 & 173 & & 1.0 & & 0.6 \\
\hline ALT (mmol/L) & 65 & & $32^{*}$ & & 27 & 109 & & 24 & & 13 & 174 & & 26 & & 16 \\
\hline $\begin{array}{l}\text { HDL } \\
\text { (mmol/L) }\end{array}$ & 62 & & 1.1 & & 0.4 & 108 & & 1.1 & & 0.3 & 171 & & 1.1 & & 0.3 \\
\hline $\mathrm{HbA1c}(\%)$ & 62 & & 5.5 & & 0.5 & 105 & & 5.4 & & 0.4 & 167 & & 5.4 & & 0.5 \\
\hline HOMA-IR & 65 & & 3.0 & & 2.9 & 108 & & 2.4 & & 2.1 & 173 & & 2.6 & & 2.6 \\
\hline $\begin{array}{l}\text { Fasting } \\
\text { insulin } \\
\text { (mU/L) }\end{array}$ & 65 & & 15 & & 14.1 & 109 & & 12.1 & & 10.5 & 174 & & 13 & & 11.1 \\
\hline $\begin{array}{l}\text { Fasting } \\
\text { glucose } \\
\text { (mmol/L) }\end{array}$ & 65 & & $4.6^{\star}$ & & 0.6 & 108 & & 4.4 & & 0.45 & 173 & & 4.4 & & 0.5 \\
\hline $\mathrm{PWV}(\mathrm{m} / \mathrm{s})$ & 54 & 7.3 & & 1.1 & & 92 & 7.1 & & 1.2 & & 146 & 7.1 & & 1.2 & \\
\hline
\end{tabular}

*Statistically significant differences between sexes $(p<0.05)$.

$\mathrm{BMI}$, body mass index; BP, blood pressure; FMI, fat mass index; HbA1c, haemoglobin 1c; HOMA, homeostatic model assessment-insulin resistance; PWV, pulse wave velocity; SAD, anterior-posterior sagittal abdominal dimension; z, z-score.

Participants with zBMI $>2.5 \mathrm{SD}$ and $>3.5 \mathrm{SD}$ had greater PWV on average than those below the cut-offs $(>2.5 \mathrm{SD}$ : 7.1 vs $6.8, \mathrm{p}=0.04 ;>3.5 \mathrm{SD}$ : 7.6 vs $7.0, \mathrm{p}=0.03)$; however, the groups overlapped substantially (see figure 2 ).

\section{DISCUSSION}

In our study of adolescents with obesity from a community sample in the UK, we found a positive association between arterial stiffness and adiposity (zBMI, FMI) including central adiposity in the form of standardised waist circumference. This association was independent of age and ethnicity. Partitioning subjects by levels of zBMI showed small differences in mean PWV by group, a finding also recently reported elsewhere ${ }^{46}$ However, it is likely that this merely reflects a positive relationship between zBMI and PWV, and there was considerable overlap of PWV between groups (seen most clearly in figure 2). Thus, our ability to classify and understand what constitutes 'severe obesity' from the perspective of contemporary pathological processes remains limited. These findings provide important information for clinicians, young people and their families to better understand long-term CVD risk of obesity within the community, but also in clinical settings.

Our study provides other novel findings. First, we found few associations between PWV and conventional 
Table 2 Univariable regression analyses of PWV on demographic, anthropometric and cardiometabolic variables

\begin{tabular}{|c|c|c|c|c|}
\hline & $\mathbf{n}$ & Coefficient & $\beta$ & p Value \\
\hline Age & 146 & 0.12 & 0.22 & $<0.01$ \\
\hline Female sex (reference male) & 146 & -0.19 & -0.08 & 0.34 \\
\hline Pubertal stage (reference late/complete) & 146 & & & \\
\hline pre/early (1\&2) & & -0.15 & 0.01 & 0.68 \\
\hline $\operatorname{mid}(3 / 4)$ & & -0.12 & 0.61 & 0.67 \\
\hline Ethnicity (reference white) & 146 & 0 & & \\
\hline Black & & 0.42 & 0.17 & 0.07 \\
\hline South Asian & & 0.67 & 0.23 & 0.01 \\
\hline Mixed other & & 0.18 & 0.05 & 0.60 \\
\hline Current smoker & & 0.27 & 0.07 & 0.43 \\
\hline Ever smoked & & 0.15 & 0.06 & 0.47 \\
\hline Height z & 146 & -0.13 & -0.11 & 0.18 \\
\hline zBMI & 146 & 0.44 & 0.20 & $0.01^{*}$ \\
\hline Fat mass index & 144 & 0.05 & 0.18 & $0.03^{*}$ \\
\hline Waist-z & 146 & 0.27 & 0.17 & $0.04^{*}$ \\
\hline SAD & 132 & 0.06 & 0.18 & $0.04^{\star}$ \\
\hline Systolic z & 146 & 0.02 & 0.02 & 0.83 \\
\hline Diastolic z & 146 & 0.08 & 0.08 & 0.33 \\
\hline Cholesterol & 146 & 0.16 & 0.12 & 0.16 \\
\hline High cholesterol vs low & 146 & -0.35 & -0.11 & 0.20 \\
\hline HDL & 142 & 0.16 & 0.03 & 0.68 \\
\hline Low HDL vs high & 142 & -0.63 & -0.17 & $0.04^{*}$ \\
\hline Triglycerides & 145 & 0.24 & 0.13 & 0.13 \\
\hline Abnormal triglycerides vs normal & 145 & 0.52 & 0.17 & $0.04^{*}$ \\
\hline ALT & 146 & 0.01 & 0.14 & 0.08 \\
\hline Fasting glucose & 146 & 0.04 & 0.02 & 0.86 \\
\hline $\mathrm{HbA1c}$ & 146 & 0.23 & 0.07 & 0.38 \\
\hline Fasting insulin & 146 & 0.01 & 0.06 & 0.50 \\
\hline Abnormal insulin vs normal & 146 & 0.17 & & 0.50 \\
\hline HOMA-IR & 146 & 0.01 & 0.01 & 0.76 \\
\hline Abnormal HOMA-IR vs normal & 146 & 0.03 & 0.01 & 0.91 \\
\hline
\end{tabular}

*Statisically significant, $p<0.05$.

BMI, body mass index; BP, blood pressure; FMI, fat mass index; HbA1c, haemoglobin 1c; HOMA, homeostatic model assessment-insulin resistance; PWV, pulse wave velocity; SAD, anterior-posterior sagittal abdominal dimension; z, z-score.

markers of cardiometabolic risk, in particular standardised BP. This is in contrast to a recent study from North America where PWV was greater in children with obesity and hypertension compared with those with obesity alone.$^{47}$ Although the prevalence of hypertension in our sample was low compared with other published samples of adolescents with obesity, ${ }^{8} 948$ the validity of non-ambulatory, isolated high BP readings recorded at single encounters that are frequently found in studies of PWV are unclear. ${ }^{49}$ There is also evidence that adiposity itself may be a driving influence on vasculature which leads to the development of hypertension. ${ }^{50-52}$ Combined with the evidence that conventional risk markers are often unstable across adolescence, ${ }^{15}$ our findings highlight the need for larger, longitudinal studies to better understand the differential relationships between adiposity, BP and arterial stiffening. We also found no association between PWV and stage of puberty, although PWV increased with age. Lack of knowledge about the effects of puberty on arterial stiffness has been identified as a key limitation of many published studies of PWV in children and adolescents with obesity. ${ }^{18} 2728$ The relationship between puberty, obesity and arterial stiffening also needs larger, longitudinal samples, as puberty is associated with the development of sexual 

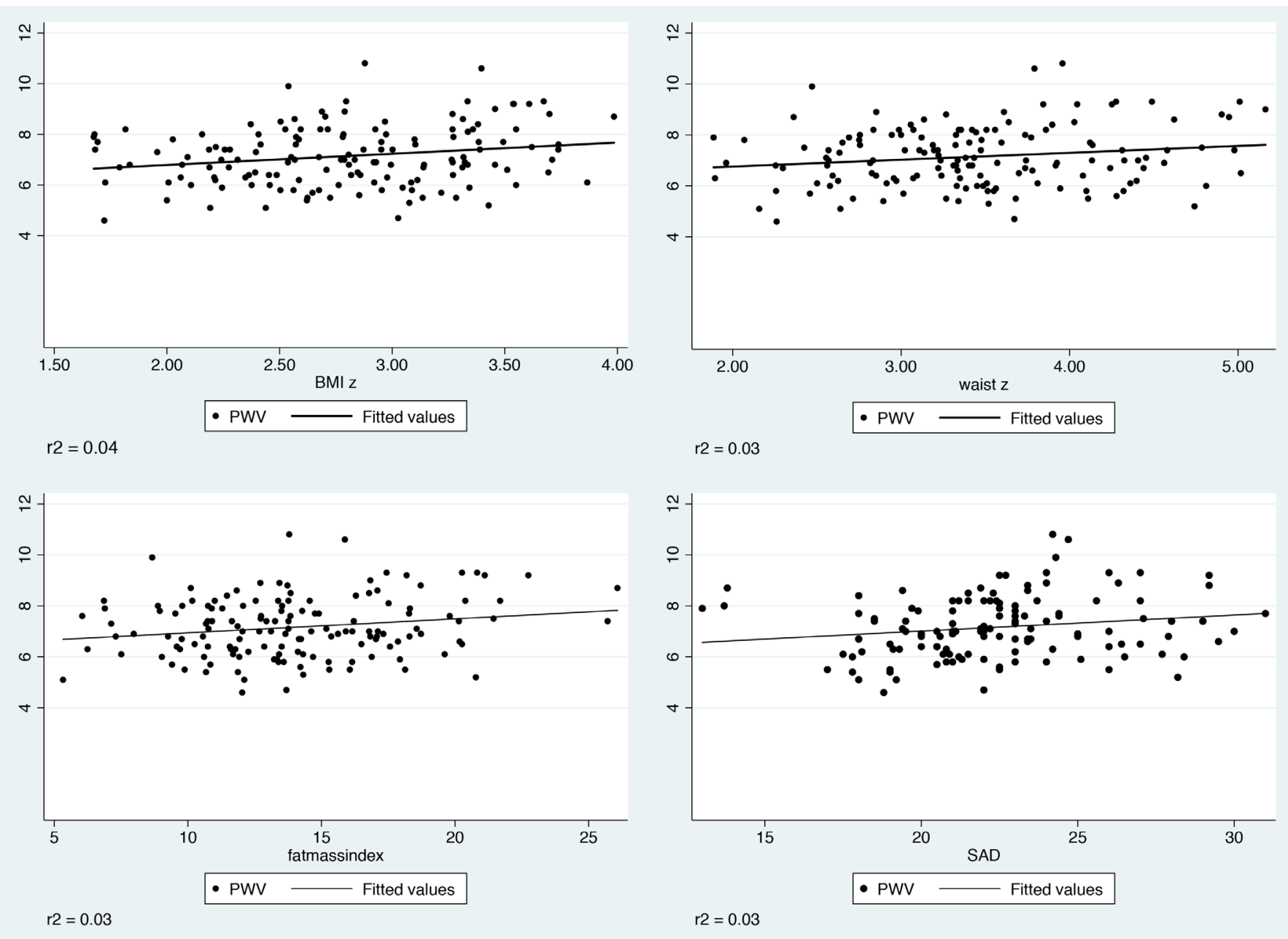

Figure 1 Scatter plot with fitted regression lines for measures of adiposity against pulse wave velocity (PWV) (m/s).

dimorphism in cardiovascular risk profiles that is not apparent earlier in childhood. Lastly, we found that South Asian adolescents had greater PWV compared with white, and our ability to describe differences in PWV between ethnicities typical of Northern European adolescent urban populations is we believe unique in the literature for the age group studied.

In our adjusted models, a 1 SD increase in BMI (ie, a unit increase in zBMI) was associated with an increase of $0.5 \mathrm{~m} / \mathrm{s}$ in $\mathrm{PWV}$, equivalent to an $0.4 \mathrm{SD}$ change in

Table 3 Multivariable analyses of pulse wave velocity on adiposity measures (adjusted for age, ethnicity, abnormal triglyceride and low HDL).

\begin{tabular}{llll}
\hline & $\mathbf{n}$ & Coefficient $(\mathbf{9 5} \% \mathbf{C l})$ & p Value \\
\hline zBMI & 145 & $0.49(0.14$ to 0.84$)$ & 0.006 \\
Waist-z & 145 & $0.26(0.01$ to 0.52$)$ & 0.04 \\
FMI & 144 & $0.05(0.01$ to 0.10$)$ & 0.002 \\
SAD & 131 & $0.05(-0.13$ to 0.10$)$ & 0.13 \\
\hline
\end{tabular}

BMI, bodymassindex; FMI, fat mass index; HDL, high-density lipoprotein; SAD, anterior-posteriorsagittal abdominal dimension.
PWV. For comparison, a meta-analysis of adult studies by Vlachopoulos et $a l^{26}$ reported that a $1 \mathrm{SD}$ increase in aortic PWV in adults led to around a $50 \%$ increase in total cardiovascular events and mortality. Similarly, Ben-Shlomo et $a l^{25}$ reported HRs of 1.35 and 1.5 for coronary heart disease and stroke, respectively associated with a 1 SD increase in the log of aortic PWV. Our data were collected by carotid-radial rather than aortic methodology, nonetheless these adult data suggest that the level of increased arterial stiffening seen in our sample is concerning, particularly for those with more extreme BMI z-scores. Encouragingly, a recent meta-analysis in adults concluded that weight loss by lifestyle change appears to reduce PWV. ${ }^{53}$

Our study has a number of strengths and limitations. Our sample was drawn from community sources and is therefore more likely to be representative of the background population of CYP with obesity from which clinical samples come. Our sample had a representative spread across age groups. Data were collected using standardised operating procedures, and in particular one operator used a rigorous procedure for measurement of PWV in all participants, thus avoiding 
PWV Above or below 2.5-z BMI

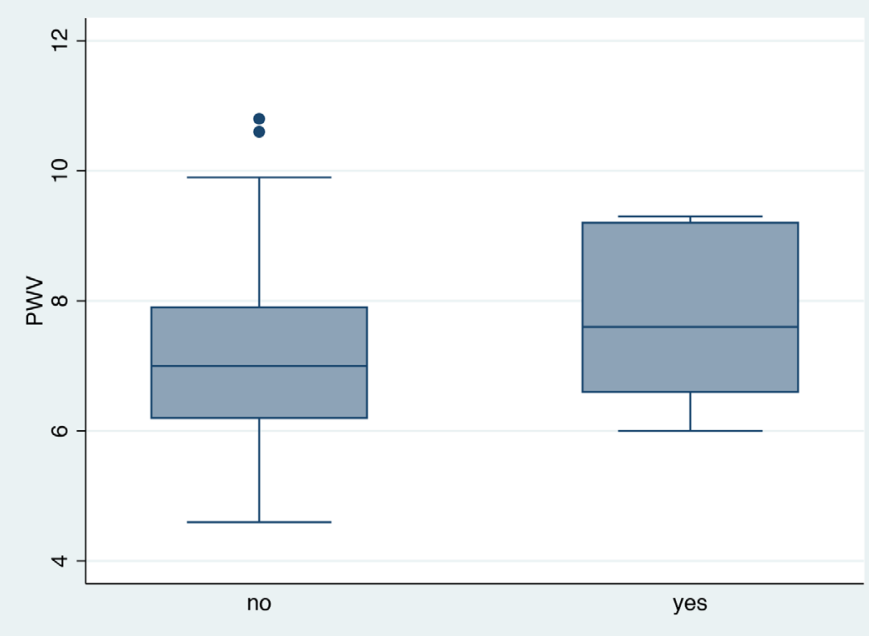

PWV Above or below 3.5-z BMI

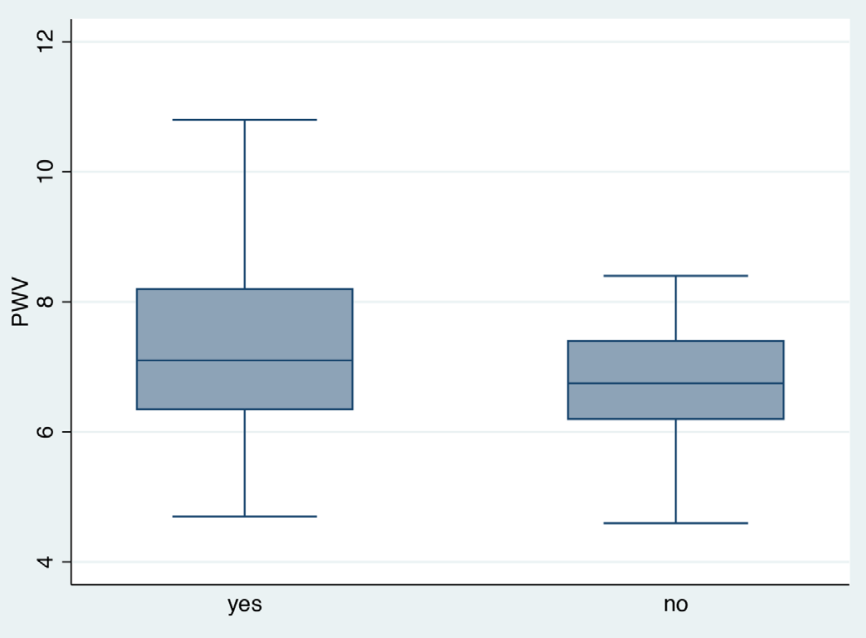

Figure 2 Box plots of distribution of pulse wave velocity (PWV) $(\mathrm{m} / \mathrm{s})$ when grouped by presence or not of severe obesity (classified by $>2.5 \mathrm{zBMI}$ and $>3.5 \mathrm{zBMI}$, respectively). Central lines are median PWV.

interoperator variability. Our protocols for measuring BP ensured ample resting time with no activity and reduced access to stress for participants and repeated measures were averaged; and moreover validity of high levels of BP found on individual encounters are unclear. ${ }^{49}$ Against that our data are cross-sectional and while they provide information on associations, they cannot be used to determine causality. There were incomplete data ( $15 \%$ of PWV). Our data come from an obesity intervention where sample size calculations were based on sufficient numbers to detect a difference between intervention and control, not for analysis of associations using cross-sectional data. Obtaining adequate power in cross-sectional studies using regression to detect small effect sizes is challenging for most studies, with a requirement for large numbers $(n>400)$ for univariable analyses. ${ }^{54}$ Our sample size was sufficiently powered (using a conventional level of power at 0.8 ) to capture associations with large and medium effects for univariable analyses, but had a power of approximately 0.4 to identify associations with small effect sizes. ${ }^{54}$ It is therefore possible that our sample size may have been insufficient to capture associations with smaller effects sizes; and this is important given that the associations we found were all small in effect. While a single operator measured PWV in all cases and data was only recorded when it met manufacturer's quality standards, only a single reading of PWV was performed per participant to avoid burden on participants, so repeated measures and analysis within individuals cannot be reported. Our measurement of PWV was also limited to the carotid-radial region (again chosen to avoid burden on participants with obesity within the main trial), and recent evidence suggests that PWV may vary by anatomical region. ${ }^{27}$ Puberty was self-assessed rather than directly rated, and disparities between trained observer and self-report of puberty have been reported ${ }^{55}$ However, we reduced misclassification bias by combining genital/breast measures with pubic hair and with menstruation in females.

\section{Conclusions}

We have demonstrated that increasing adiposity is associated with greater PWV, a proxy for arterial stiffening, within a group of adolescents with obesity. This is concerning for the future CVD risk for a current generation with a high prevalence of obesity, and highlights the importance of research for successful interventions for obesity in young people. Caution must be exercised in interpreting the significance of conventional cardiometabolic markers such as hypertension and dyslipidemia, especially when considering pharmacological treatments for them. The long-term effect of changes in adiposity on PWV in adolescence remains unclear and larger, longitudinal studies are needed to investigate this further.

Acknowledgements The data come from an adolescent obesity intervention funded separately to this work by the National Institute for Health Research, UK. The authors would like to thank the young people and their families who took part in the original study.

Contributors LH produced the first draft of the paper, co-conceived the study and collected the data. SK co-supervised the study and data collection, contributed to the final draft. IW co-supervised the study and data collection, contributed to the final draft. TJC assisted in the analysis and contributed to the final draft. JD co-supervised the data collection and contributed to the final draft. RV was main supervisor for the study, co-supervised the study and contributed to the final draft. All authors approved the final manuscript as submitted and agree to be accountable for all aspects of the work.

Competing interests None declared.

Ethics approval Central London Ethics Committee.

Provenance and peer review Not commissioned; externally peer reviewed.

Open Access This is an Open Access article distributed in accordance with the Creative Commons Attribution Non Commercial (CC BY-NC 4.0) license, which permits others to distribute, remix, adapt, build upon this work non-commercially, and license their derivative works on different terms, provided the original work is 
properly cited and the use is non-commercial. See: http://creativecommons.org/ licenses/by-nc/4.0/

(C) Article author(s) (or their employer(s) unless otherwise stated in the text of the article) 2017. All rights reserved. No commercial use is permitted unless otherwise expressly granted.

\section{REFERENCES}

1. Ng M, Fleming T, Robinson $\mathrm{M}$, et al. Global, regional, and national prevalence of overweight and obesity in children and adults during 1980-2013: a systematic analysis for the global burden of disease study 2013. Lancet 2014;384:766-81.

2. Ogden CL, Flegal KM, Carroll MD, et al. Prevalence and trends in overweight among US children and adolescents, 1999-2000. JAMA 2002;288:1728-32.

3. Viner RM, White B, Barrett T, et al. Assessment of childhood obesity in secondary care: osca consensus statement. Arch Dis Child Educ Pract Ed 2012;97:98-105.

4. Barlow SE; Expert Committee. Expert committee recommendations regarding the prevention, assessment, and treatment of child and adolescent overweight and obesity: summary report. Pediatrics 2007;120 Suppl 4:S164-S192.

5. Rolland-Cachera MF. Childhood obesity: current definitions and recommendations for their use. Int J Pediatr Obes 2011;6:325-31.

6. Cole TJ, Bellizzi MC, Flegal KM, et al. Establishing a standard definition for child overweight and obesity worldwide: international survey. BMJ 2000;320:1240.

7. Freedman DS, Mei Z, Srinivasan SR, et al. Cardiovascular risk factors and excess adiposity among overweight children and adolescents: the Bogalusa Heart Study. J Pediatr 2007;150:12-17.

8. Weiss R, Dziura J, Burgert TS, et al. Obesity and the metabolic syndrome in Children and Adolescents. N Engl J Med Overseas Ed 2004;350:2362-74.

9. van Emmerik NM, Renders $\mathrm{CM}$, van de Veer $\mathrm{M}$, et al. High cardiovascular risk in severely obese young children and adolescents. Arch Dis Child 2012;97:818-21.

10. Webber LS, Harsha DW, Phillips GT, et al. Cardiovascular risk factors in Hispanic, white, and black children: the Brooks County and Bogalusa Heart studies. Am J Epidemiol 1991;133:704-14.

11. Srinivasan SR, Bao W, Wattigney WA, et al. Adolescent overweight is associated with adult overweight and related multiple cardiovascular risk factors: the Bogalusa Heart Study. Metabolism 1996;45:235-40.

12. Zimmet P, Alberti KG, Kaufman F, et al. The metabolic syndrome in children and adolescents - an IDF consensus report. Pediatr Diabetes 2007;8:299-306.

13. Viner RM, White B, Barrett T, et al. Assessment of childhood obesity in secondary care: osca consensus statement. Arch Dis Child Educ Pract Ed 2012;97:98-105.

14. Steinberger J, Daniels SR, Eckel RH, et al. Progress and challenges in metabolic syndrome in children and adolescents: a scientific statement from the american Heart Association Atherosclerosis, hypertension, and obesity in the Young Committee of the Council on Cardiovascular Disease in the young; Council on Cardiovascular Nursing; and Council on Nutrition, Physical Activity, and metabolism. Circulation 2009;119:628-.

15. Goodman E, Daniels SR, Meigs JB, et al. Instability in the diagnosis of metabolic syndrome in adolescents. Circulation 2007:115:2316-22.

16. Hudson L, Viner RM. Obesity in children and adolescents. BMJ: British Medical Journal 2012:345.

17. Ayer J, Steinbeck K. Placing the cardiovascular risk of childhood obesity in perspective. Int J Obes 2010;34:4-5.

18. Cote AT, Harris KC, Panagiotopoulos C, et al. Childhood obesity and cardiovascular dysfunction. J Am Coll Cardiol 2013;62:1309-19.

19. McGill HC, McMahan CA, Herderick EE, et al. Origin of atherosclerosis in childhood and adolescence. Am J Clin Nutr 2000;72:1307S.

20. Laurent S, Cockcroft J, Van Bortel L, et al. Expert consensus document on arterial stiffness: methodological issues and clinical applications. Eur Heart J 2006;27:2588-605.

21. Nichols WW. Clinical measurement of arterial stiffness obtained from noninvasive pressure waveforms. Am J Hypertens 2005;18:3-10.

22. Anderson TJ, Phillips SA. Assessment and prognosis of peripheral artery measures of vascular function. Prog Cardiovasc Dis 2015;57.

23. Donald AE, Charakida M, Falaschetti E, et al. Determinants of vascular phenotype in a large childhood population: the Avon Longitudinal Study of Parents and Children (ALSPAC). Eur Heart J 2010;31:1502-10.
24. McLeod AL, Uren NG, Wilkinson IB, et al. Non-invasive measures of pulse wave velocity correlate with coronary arterial plaque load in humans. J Hypertens 2004;22:363-8.

25. Ben-Shlomo Y, Spears M, Boustred C, et al. Aortic pulse wave velocity improves cardiovascular event prediction: an individual participant meta-analysis of prospective observational data from 17,635 subjects. J Am Coll Cardiol 2014:63:636-46.

26. Vlachopoulos C, Aznaouridis K, Stefanadis C. Prediction of cardiovascular events and all-cause mortality with arterial stiffness: a systematic review and meta-analysis. J Am Coll Cardiol 2010;55:1318-27.

27. Hudson LD, Rapala A, Khan T, et al. Evidence for contemporary arterial stiffening in obese children and adolescents using pulse wave velocity: a systematic review and meta-analysis. Atherosclerosis 2015;241:376-86.

28. Cote AT, Phillips AA, Harris KC, et al. Obesity and arterial stiffness in children: systematic review and meta-analysis. Arteriosclerosis. thrombosis, and vascular biology 2015;35:1038-44.

29. Pandit D, Kinare A, Chiplonkar S, et al. Carotid arterial stiffness in overweight and obese indian children. J Pediatr Endocrinol Metab 2011;24:97-102.

30. Pandit DS, Khadilkar AV, Chiplonkar SA, et al. Arterial stiffness in obese children: role of adiposity and physical activity. Indian J Endocrinol Metab 2014:18:70-6.

31. Arnberg K, Larnkjær A, Michaelsen KF, et al. Central adiposity and protein intake are associated with arterial stiffness in overweight children. J Nutr 2012;142:878-85.

32. Kudo U, Takahashi I, Matsuzaka M, et al. Influence of obesity on blood pressure and arterial stiffness in the early teens. Obes Res Clin Pract 2013;7:e211-e217.

33. Lee JW, Lee DC, Im JA, J-a I, et al. Insulin resistance is associated with arterial stiffness independent of obesity in male adolescents. Hypertens Res 2007;30:5-11.

34. Miyai N, Arita M, Miyashita K, et al. The influence of obesity and metabolic risk variables on brachial-ankle pulse wave velocity in healthy adolescents. J Hum Hypertens 2009;23:444-50.

35. Dangardt F, Osika W, Volkmann R, et al. Obese children show increased intimal wall thickness and decreased pulse wave velocity. Clin Physiol Funct Imaging 2008;28:287-93.

36. Gungor N, Thompson T, Sutton-Tyrrell K, et al. Early signs of cardiovascular disease in youth with obesity and type 2 diabetes. Diabetes Care 2005;28:1219-21.

37. Christie D, Hudson LD, Kinra S, et al. Assessing the efficacy of the Healthy Eating and Lifestyle Programme (HELP) compared with enhanced standard care of the obese adolescent in the community: study protocol for a randomized controlled trial. Arch Dis Child 2017;102:695-701. Epub 2017 Jul 7.

38. Taylor SJ, Whincup PH, Hindmarsh PC, et al. Performance of a new pubertal self-assessment questionnaire: a preliminary study. Paediatr Perinat Epidemiol 2001;15:88-94.

39. Haroun D, Croker H, Viner RM, et al. Validation of BIA in obese children and adolescents and re-evaluation in a longitudinal study. Obesity 2009;17:2245-50.

40. McCarthy HD, Jarrett KV, Crawley HF. The development of waist circumference percentiles in british children aged 5.0-16.9 y. Eur $J$ Clin Nutr 2001;55:902-7.

41. Kahn HS. Choosing an index for abdominal obesity: an opportunity for epidemiologic clarification. J Clin Epidemiol 1993;46:491-4

42. Cole TJ, Freeman JV, Preece MA. Body mass index reference curves for the UK, 1990. Arch Dis Child 1995;73:25-9.

43. Cole TJ. The LMS method for constructing normalized growth standards. Eur J Clin Nutr 1990;44:45-60.

44. Jackson LV, Thalange NK, Cole TJ. Blood pressure centiles for Great Britain. Arch Dis Child 2007;92:298-303.

45. Cohen J. Statistical power analysis for the behavioral sciences (revised ed). Hillsdale, NJ: Erlbaum, 1988.

46. Shah AS, Dolan LM, Khoury PR, et al. Severe obesity in adolescents and young adults is associated with Sub-Clinical cardiac and vascular changes. The Journal of clinical endocrinology and metabolism 2015;100:2751-7. jc20144562.

47. Kulsum-Mecci N, Goss C, Kozel BA, et al. Effects of obesity and hypertension on pulse wave velocity in Children. J Clin Hypertens 2017;19.

48. Friedemann C, Heneghan C, Mahtani K, et al. Cardiovascular disease risk in healthy children and its association with body mass index: systematic review and meta-analysis. BMJ 2012;345:e4759.

49. Urbina E, Alpert B, Flynn J, et al. Ambulatory blood pressure monitoring in children and adolescents: recommendations for 
standard assessment: a scientific statement from the american Heart Association Atherosclerosis, hypertension, and obesity in Youth Committee of the council on cardiovascular disease in the young and the council for high blood pressure research. Hypertension 2008;52:433-51.

50. Weisbrod RM, Shiang T, Al Sayah L, et al. Arterial stiffening precedes systolic hypertension in diet-induced obesity. Hypertension 2013;62:1105-10.

51. Kaess BM, Rong J, Larson MG, et al. Aortic stiffness, blood pressure progression, and incident hypertension. JAMA 2012;308:875-81.
52. Rahmouni K. Obesity-associated hypertension: recent progress in deciphering the pathogenesis. Hypertension 2014;64:215-21.

53. Petersen KS, Blanch N, Keogh JB, et al. Effect of weight loss on pulse wave velocity: systematic review and meta-analysis. Arterioscler Thromb Vasc Biol 2015;35:243-52.

54. Miles J, Shevlin M. Applying regression and correlation: a guide for students and researchers. Sage 2001.

55. Dorn LD, Biro FM. Puberty and its Measurement: a Decade in Review. Journal of Research on Adolescence 2011;21:180-95. 